\title{
MÉTODOS DE PESQUISA EM AVALIAÇÃO DE TECNOLOGIA EM SAÚDE
}

\author{
Marcelo Eidi NITA', Silvia Regina SECOLI², Moacyr NOBRE ${ }^{3}$ e Suzane Kioko ONO-NITA ${ }^{1}$
}

RESUMO - Existe, atualmente, grande demanda para se aumentar a eficiência do ato médico e isto pode ser alcançado através de pesquisas de avaliação de tecnologia em saúde, a ATS. Esta visa, por um lado, determinar a melhor evidência de eficácia ou efetividade de um dado tratamento em saúde, por outro, determinar os custos associados com tal tratamento médico. Somente as alternativas com custo e efetividade comprovados, ou seja, que sejam eficientes, serão adotados doravante nos hospitais e sistemas de saúde público e privados. Exemplo são os custos crescentes de tratamentos com biológicos, por exemplo, em doença inflamatória intestinal ou hepatites virais, ou mesmo em oncologia. Há necessidade de se desenvolver pesquisas em ATS para a identificação não somente dos tratamentos que funcionam dos que não funcionam, mas também se os custos a eles associados compensam o seu uso. Este artigo introduz esta terminologia e os métodos para se desenvolver esses estudos.

DESCRITORES - Pesquisa biomédica. Medicina baseada em evidências. Economia da saúde. Avaliação de medicamentos. Avaliação de programas e projetos de saúde.

\section{INTRODUÇÃO}

O nosso sistema de saúde, baseado em conceitos de universalidade e equidade da assistência à saúde teve um grande avanço com a criação do Sistema Único de Saúde - SUS. No entanto, carências neste modelo culminaram com o desenvolvimento da saúde suplementar ou privado. Ainda assim, há uma ênfase na melhora da eficiência do sistema de saúde, o que tem criado a necessidade explícita de realizar quantificação e justificativa de benefícios e custos associados aos serviços de saúde e terapias específicas, no sentido de haver decisões clínicas mais eficientes. Desta forma, observa-se no contexto mundial um crescimento expressivo de estudos de avaliação de tecnologias em que são utilizadas técnicas sofisticadas para comparar distintas alternativas de tratamentos.

A avaliação de tecnologia em saúde (ATS) pode ser entendida como a pesquisa sistemática da melhor evidência disponível da eficácia ou de efetividade de uma tecnologia em saúde, e dos custos relacionados a ela. O intuito da ATS é permitir que sistemas ou organizações de saúde, como por exemplo, hospitais e clinicas possam aumentar a qualidade e o bem estar do paciente e otimizar a relação de custo-efetividade, ou seja, a eficiência de produtos para saúde (definições na Figura 1). As tecnologias em saúde são definidas como medicamentos, procedimentos médicos, equipamentos e, até mesmo, programas de cuidados

\begin{tabular}{|l|l|}
\hline Eficácia & $\begin{array}{l}\text { Estudo para determinar os benefícios de uma nova tecnologia } \\
\text { utilizada em condições ideais ou experimentais. O conceito, } \\
\text { geralmente, refere-se a resultados obtidos a partir de estudos } \\
\text { clínicos controlados e randomizados (ECCR) }\end{array}$ \\
\hline Efetividade & $\begin{array}{l}\text { Estudo para determinar os benefícios de uma nova tecnologia } \\
\text { utilizada em condições habituais ou do dia-a-dia. O conceito, } \\
\text { geralmente, refere-se a resultados obtidos a partir de estudos } \\
\text { clínicos pragmáticos ou práticos, estudos observacionais } \\
\text { (prospectivos ou retrospectivos) ou de revisão de séries de } \\
\text { casos (obtidos de prontuários médicos ou banco de dados, } \\
\text { como o Datasus) }\end{array}$ \\
\hline Eficiência & $\begin{array}{l}\text { Conceito econômico em que se busca o maior benefício com } \\
\text { o menor custo possível. O conceito, geralmente refere-se aos } \\
\text { estudos de custo-efetividade ou custo-utilidade }\end{array}$ \\
\hline
\end{tabular}

Fonte: modificado de Nita ME, et al.(2009)

FIGURA 1. Definições utilizadas em estudos de avaliação de tecnologia em saúde - ATS

para saúde, como por exemplo, aconselhamento nutricional.

Em sua essência, a ATS pode ser vista pela perspectiva clinica e econômica. Dada a complexidade dessa "união", especialmente porque cada uma das perspectivas apresenta particularidades no que concerne a terminologias e técnicas, foi essencial o envolvimento de outras áreas do conhecimento para a realização dos estudos de ATS.

No que tange ao aspecto clínico, a ATS possibilita a integração da ciência na prática de cuidados à saúde, ou seja, adota a medicina baseada em evidência (MBE), fruto esta do conhecimento de disciplinas como a epidemiologia clínica, bioestatística e saúde pública.

'Departamento de Gastroenterologia, Faculdade de Medicina, Universidade de São Paulo (USP); ${ }^{2}$ sscola de Enfermagem USP; ${ }^{3}$ Unidade de Epidemiologia Clínica, Instituto do Coração, Hospital das Clínicas, Faculdade de Medicina USP, São Paulo, SP.

Correspondência: Dr. Marcelo E. Nita - Rua Caraíbas, 326 - apt.181 - Perdizes - 05020-000 - SÃO PAULO, SP. E-mail: marcelo_nita@uol.com.br 
A MBE permite aliar o rigor metodológico dessas áreas de conhecimento à experiência clínica do profissional da saúde no manejo diário do paciente.

Pelo lado econômico, a ATS pode ser vista como a otimização do benefício clínico em relação aos recursos, inclusive monetários, empregados nos cuidados à saúde, ou seja, a economia da saúde, produto este do conjunto de conhecimento de áreas da saúde, de gestão e da economia. A economia da saúde permite identificar os recursos associados aos cuidados em saúde, visando a melhor alocação destes entre as diferentes tecnologias que competem entre si.

Em suma, a agregação da análise dos benefícios clínicos e dos custos da prestação do serviço em saúde permite, por meio da realização dos estudos de custo-efetividade ou utilidade, que as tomadas de decisão, independente do nível da esfera decisória, sejam feitas de modo objetivo, transparente e, principalmente, fundamentada em parâmetros que agreguem valor e sejam reconhecidos entre profissionais da saúde (médicos, enfermeiros, nutricionistas, farmacêuticos, fisioterapeutas, dentistas, etc), entre pacientes e entre gestores de serviços de saúde.

Tendo em vista a importância da ATS como área de conhecimento emergente e determinante da nossa prática diária, o escopo deste é destacar conceitos teóricos que possam subsidiar a realização de pesquisas nesta área e auxiliar na tomadas de decisões na prática clínica.

\section{Aspectos essenciais da pesquisa em ATS}

Nosso objetivo então é a elaboração de investigações científicas de qualidade sobre a eficácia e efetividade, bem como, acerca da eficiência, que tragam impacto prático no cenário brasileiro no que tange a melhoria contínua do nível de saúde.

A avaliação da eficácia, efetividade e eficiência encontramse atreladas ao desenvolvimento de projetos de pesquisas clínicas, revisões sistemáticas da literatura e metanálise, modelos de decisão clínica ou análises econômicas em saúde. Todavia, a elaboração desses estudos requer inicialmente a apresentação de uma pergunta de pesquisa, ou seja, uma idéia ou questão clínica. De fato, todo projeto de pesquisa deve iniciar com uma pergunta de pesquisa que apresente características FINERS ("feasible, interesting, novel, ethical, relevant and specific") para garantir que a pesquisa aborde temas de aplicabilidade no dia-a-dia, assim como o uso do PICO ("patient, intervention, control and outcomes") que assegure que a pergunta apresente os ingredientes sine qua non para a sua realização. A partir da definição da pergunta da pesquisa é possível vislumbrar as melhores opções de métodos e técnicas a serem descritas no nosso protocolo de pesquisa.

A seguir listamos, a titulo de exemplo, algumas perguntas que podem ser feitas e a relação que pode ser estabelecida com os tipos de estudo:

1) Este tipo de tratamento clínico (tecnologia) reduz a mortalidade quando comparado com o tratamento endoscópico em pacientes com diagnóstico de úlcera péptica com sangramento ativo?
2) Qual a melhor opção entre os diversos tipos de intervenção cirúrgica para o paciente com câncer gástrico precoce, em termos de sobrevida em 5 anos?

3) Qual será o prognóstico do paciente com diagnóstico recém estabelecido de doença de Crohn?

4) Entre as várias opções de tratamento clínico para a erradicação do Helicobacter pylori, qual apresenta a melhor relação de custo-efetividade?

5) Qual o tratamento clínico com melhor impacto na qualidade de vida do paciente com hepatite crônica por vírus C?

Para as questões 1 e 2 , provavelmente, as melhores alternativas de pesquisa sejam o estudo clínico controlado e randomizado (ECCR) e a revisão sistemática com metanálise. A questão 3 pode ser respondida por estudos de coorte; a 4 por estudos de economia da saúde. A última pergunta (5) remete à necessidade de um tipo especial de estudo denominado "patient reported outcomes" (PRO), que no caso do exemplo possibilitará a avaliação da qualidade de vida relacionado à saúde ("health related quality of life" - HRQOL) do paciente com hepatite C. Desse modo, é importante enfatizar que nem toda investigação necessita de um ECCR, dito como padrãoouro da MBE. O melhor desenho de estudo irá depender da formulação da pergunta da pesquisa.

\section{Desenhos de pesquisa clínica, de efetividade e econômica}

Uma vez estabelecidos a pergunta da pesquisa temos, pois, de associar esta aos diferentes métodos e técnicas envolvidas nos desenho de estudos. Inicialmente, vamos verificar se os pesquisadores realizam uma intervenção que expõe o individuo a alguma tecnologia de saúde como, por exemplo, um novo medicamento, ou novo tipo de intervenção endoscópica, ou mesmo novo tipo de curativo para auxiliar a cicatrização de feridas. Caso haja, o estudo é denominado experimental, caso contrário, trata-se de estudo observacional.

Desse modo, estudos clínicos, cuja participação do pesquisador restringe-se a observar os eventos, sem desempenhar um papel ativo, recebem a denominação de observacional. São exemplos desse tipo de estudo a coorte e o caso-controle, que se destinam, na maioria das vezes, a avaliar o risco de um dado desfecho.

Reparem que o estudo experimental é um tipo especial de coorte em que a seleção dos grupos de tratamento, natureza das intervenções e manejo durante o seguimento e aferição dos resultados são determinados pelo pesquisador - são os chamados ensaios clínicos.

No estudo experimental, a classificação em randomizado ou não, depende da aleatoriedade da alocação do tratamento ou da tecnologia. No caso de ser aleatória, o estudo recebe a denominação de estudo clínico randomizado; caso contrário, será estudo clínico não-randomizado. Esses estudos podem, ainda, apresentar um grupo comparador ou controle. Estudos experimentais são, frequentemente, de dois braços, sendo um controle, ocasião em que serão chamados estudos clínicos controlados e randomizados (ECCR). 
Segundo Nobre ${ }^{(11)}$ há, basicamente, três tipos principais de desenhos de estudo ou pesquisa: transversal, caso-controle e coorte sua variante mais conhecida o $\mathbf{E C C R}$, cujas características são descritas na Figura 2.

\begin{tabular}{|l|l|}
\hline Tipo desenho & Características \\
\hline Transversal & $\begin{array}{l}\text { A coleta de dados de uma amostra populacional dos grupos } \\
\text { em estudo e controle em um mesmo momento da evolução da } \\
\text { doença. As variáveis de exposição ("exposure variable") e desfechos } \\
\text { ("outcome endpoint") são identificadas ao mesmo tempo }\end{array}$ \\
\hline Caso-controle & $\begin{array}{l}\text { Casos com critérios estabelecidos e comparados com controles. } \\
\text { A variável de desfecho ("outcome endpoint") é identificada } \\
\text { no início da pesquisa e se busca identificar qual é a variável } \\
\text { exposição ("exposure variable") } \\
\text { Desejável o pareamento de comorbidades e fatores de confusão }\end{array}$ \\
\hline Coorte & $\begin{array}{l}\text { Grupo de pessoas com alguma característica bem definida e } \\
\text { seguida durante o tempo. A variável de exposição ("exposure } \\
\text { variable") é identificada no início da pesquisa e se busca } \\
\text { identificar a variável desfecho ("outcome endpoint") } \\
\text { Gera dados de incidência e medidas de risco }\end{array}$ \\
\hline $\begin{array}{l}\text { Ensaio clinico controlado } \\
\text { randomizado - ECCR }\end{array}$ & $\begin{array}{l}\text { Pessoas distribuídas por sorteio em grupos de intervenção e } \\
\text { controle, seguidas durante o tempo } \\
\text { Gera dados de incidência e medidas de risco }\end{array}$ \\
\hline
\end{tabular}

Fonte: modificado de Nobre MRC, Bernardo W. (2006) e Nita ME, et al. (2009)

FIGURA 2. Tipos e características de desenhos de estudos

Há, ainda, os chamados estudos de síntese do conhecimento, representados pela revisão sistemática (RS) e metanálise (MA). No âmbito atual, ambos representam avanço importante entre os métodos científicos empregados na saúde e em especial na ATS. Possivelmente, este será o primeiro passo em um estudo de ATS, ou seja, só vale a pena estudar a relação de custo e benefício de uma tecnologia se esta for comprovadamente eficaz.

A RS é um processo objetivo e criterioso de pesquisa que utiliza método de busca compreensiva e extensiva da evidência clínica, sendo totalmente distinto das revisões narrativas tradicionais. A MA é método estatístico que permite a agregação de diversos estudos identificados na RS permitindo, então, aumentar a precisão das estimativas dos desfechos ("outcome variables") clínicos analisados. Esses métodos não são restritos a ECCR e podem ser empregados, com alguns cuidados, nos estudos observacionais.

A análise ou modelo de decisão representa importante avanço metodológico que permite lidar de maneira estruturada com as incertezas inerentes à prática dos cuidados em saúde. Trata-se de método gráfico, no qual as alternativas ou tecnologias estudadas são colocadas lado a lado e comparadas diretamente. Para cada uma dessas "alternativas" são elencadas as probabilidades de ocorrência, por exemplo, do desfecho, as quais, geralmente, são oriundas de resultados de estudos clínicos ou observacionais. Desse modo, o modelo permite análise quantitativa de problemas clínicos e, por esta razão, são usadas, frequentemente, nos estudos de avaliação econômica em saúde.

Todos os desenhos de estudos apresentados podem subsidiar a construção de avaliações econômicas como, por exemplo, análise de custo-efetividade e custo-utilidade. Este tipo de "avaliação" se faz mister em condições onde há limitação de recursos disponíveis frente a uma demanda por qualidade de vida e saúde crescentes da população. Hoje são pesquisas obrigatórias quando há solicitação de inclusão de inovações tecnológicas, como novos medicamentos ou novos equipamentos médicos, para o acesso e uso disseminado pela comunicada de profissionais da saúde.

$\mathrm{Na}$ prática, quem deve ser o responsável pela decisão de incorporação de nova tecnologia de saúde no âmbito do SUS ou do sistema privado? O profissional de saúde, o gestor de saúde, do produtor do insumo ou o paciente? Talvez o melhor modelo para responder esta pergunta venha do Reino Unido que integra a opinião de todos os interessados de maneira transparente através do "National Institute for Health and Cinical Excellence" (NICE).

Assim, os profissionais da saúde e a academia devem se envolver de maneira crescente na realização destas pesquisas. Num futuro muito próximo, as ATS serão os principais determinantes da maneira como será realizada a assistência à saúde.

\section{CONCLUSÃO}

Hoje, existe claro avanço nos projetos de pesquisa no Brasil alinhados ao desenvolvimento científico internacional. No entanto, há ainda muito a fazer nesta longa caminhada da ATS. A pesquisa aplicada à prática diária, com identificação de tecnologias, cuja eficácia, efetividade e eficiência sejam comprovadas, seguramente, levarão a otimização dos benefícios com custos compatíveis. Em última análise, poderão viabilizar o modelo de assistência à saúde idealizada na Constituição Brasileira. Desse modo, as pesquisas clínicas realizadas nos grandes centros universitários e hospitalares devem responder questões clínicas e econômicas relevantes e determinantes à prática da assistência da saúde. Assim, torna-se um pré-requisito o pleno domínio das inúmeras opções metodológicas tanto para sua execução tanto quanto para a sua leitura e aplicação. 
Nita ME, Secoli SR, Nobre M, Ono-Nita SK. Health technology assessment: research methodology. Arq Gastroenterol. 2009;46(4):252-5.

ABSTRACT - Currently it is expected a higher efficiency of health care and this can be achieved by health technology assessment . This aims, for one side, to determine the best evidence of efficacy or effectiveness of a given treatment, and, on the other side, to determine the costs associated with this treatment. Only cost-effective alternatives, in other words, efficients, should be adopted in hospitals or public or private health care system. For instances, the increasing costs of biologics treatments in inflammatory bowel disease or hepatology or oncology. There is a need to increase the number of health technology assessment research not only to identify those treatment that works from those does not, but also whether the costs associated with each treatment compensate its use. This young researcher forum article introduce the concepts and basic methods used in health technology assessment studies.

HEADINGS - Biomedical research. Evidence-based medicine. Health economics. Drug evaluation. Program evaluation.

\section{LITERATURA ADICIONAL}

1. Bocchi EA, Marin-Neto JA. Aplicação dos princípios da medicina baseada em evidências em programas de avaliação de procedimentos e medicamentos de alto custo. Arq Bras Cardiol. 2001;76:339-42.

2. Grimes DA, Schulz KF. An overview of clinical research: the lay of the land. Lancet. 2002:359:57-61

3. Grimes DA, Schulz KF. Cohort studies: marching towards outcomes. Lancet. 2002:359:341-5.

4. Grimes DA, Schulz KF. Descriptive studies: what they can and cannot do. Lancet. 2002:359:145-9.

5. Hulley SB, Cummings SR, Browner WS, Grady DG, Newman TB. Designing clinical research. 3rd edition. Philadelphia: Wolters Klumer Health/Lippincott Williams \& Wilkins; 2007.

6. Hunink MG, Glasziou P. Decision making in health and medicine, integrating evidence and values. Cambridge: Cambridge University Press; 2001.

7. Inadomi JM. Decision analysis and economic modelling: a primer. Eur J Gastroenterol Hepatol. 2004;16:535-42.

8. Maluf-Filho F, Nobre M, Nita ME. Análise crítica dos desenhos de pesquisa epidemiológica e clínica. In: Nita ME, Campino AC, Secoli S, Sarti FM, Nobre M, Costa AM, Ono-Nita SK, Carrilho FJ. Avaliação de tecnologias em saúde: evidência clínica, análise econômica e análise de decisão. Porto Alegre: Artmed; 2009. p.72-82.
9. Nita ME, Campino AC, Secoli S, Sarti FM, Nobre M, Costa AM, Ono-Nita SK, Carrilho FJ. Avaliação de tecnologias em saúde: evidência clínica, análise econômica e análise de decisão. Porto Alegre: Artmed; 2009.

10. Nita ME, Nobre M, Secoli S, Costa AM, Ono-Nita SK, Sarti FM, Carrilho FJ. Campino AC. Visão geral dos métodos em avaliação de tecnologias em saúde. In: Nita ME, Campino AC, Secoli S, Sarti FM, Nobre M, Costa AM, Ono-Nita SK, Carrilho FJ. Avaliação de tecnologias em saúde: evidência clínica, análise econômica e análise de decisão. Porto Alegre: Artmed; 2009. p.21-30.

11. Nobre MRC, Bernardo W, Jatene FB. A prática clínica baseada em evidências Parte 1-Questões clínicas bem construídas. Rev Assoc Med Brasil. 2003;49:445-9.

12. Nobre MRC, Bernardo W. Prática clínica baseada em evidência. São Paulo: Elservier; 2006.

13. Petitti DB. Meta-analysis, decision analysis and cost-effectiveness analysis. New York: Oxford University Press; 2000.

14. Rubenstein JH, Inadomi JM. Evidence based-medicine (EBM) in practice: applying results of cost-effectiveness analyses. Am J Gastroenterol. 2006;101:1169-71.

15. Sackett DL, Straus S, Richardson S, Rosenberg W, Haynes RB. Evidence-based medicine: how to practice and teach EBM. 2nd. ed. London: Churchill Livingstone; 2000 .

16. Secoli SR, Padilha KG, Litvoc J, Maeda ST. Farmaeconomia: perspectiva emergente no processo de tomada de decisão. Ciênc Saúde Coletiva. 2005;10(supl):287-96.

17. Sonnenberg A. Decision analysis in clinical gastroenterology. Am J Gastroenterol. 2004;99:163-9. Erratum in: Am J Gastroenterol. 2004;99:398. 